\title{
AFFINELY INFINITELY DIVISIBLE DISTRIBUTIONS AND THE EMBEDDING PROBLEM
}

\author{
S. G. DAni AND Klaus SchmidT
}

\begin{abstract}
Let $A$ be a locally compact abelian group and let $\mu$ be a probability measure on $A$. A probability measure $\lambda$ on $A$ is an affine $k$-th root of $\mu$ if there exists a continuous automorphism $\rho$ of $A$ such that $\rho^{k}=I$ (the identity transformation) and $\lambda * \rho(\lambda) * \rho^{2}(\lambda) * \cdots * \rho^{k-1}(\lambda)=\mu$, and $\mu$ is affinely infinitely divisible if it has affine $k$-th roots for all orders. Clearly every infinitely divisible probability measure is affinely infinitely divisible. In this paper we prove the converse for connected abelian Lie groups: Every affinely infinitely divisible probability measure on a connected abelian Lie group $A$ is infinitely divisible.

If $G$ is a locally compact group, $A$ a closed abelian subgroup of $G$, and $\mu$ a probability measure on $G$ which is supported on $A$ and infinitely divisible on $G$, we give sufficient conditions which ensure that $\mu$ is infinitely divisible on $A$.
\end{abstract}

\section{Introduction}

Let $A$ be a locally compact abelian group and let $\mu$ be a probability measure on $A$. A probability measure $\lambda$ on $A$ is said to be an affine $k$-th root of $\mu$ if there exists a continuous automorphism $\rho$ of $A$ such that $\rho^{k}=I$ (the identity transformation) and

$$
\lambda * \rho(\lambda) * \rho^{2}(\lambda) * \cdots * \rho^{k-1}(\lambda)=\mu .
$$

We recall that a probability measure is said to be infinitely divisible if it has convolution roots of all orders (namely $k$-th roots for all natural numbers $k$ ). In analogy with this we say that a probability measure $\mu$ on $A$ is affinely infinitely divisible (on $A$ ) if it has affine $k$-th roots for all natural numbers $k$. Clearly every infinitely divisible probability measure is affinely infinitely divisible. Here we prove that the converse is also true for connected abelian Lie groups:

Theorem 1.1. Every affinely infinitely divisible probability measure on a connected abelian Lie group $A$ is infinitely divisible on $A$.

Let $A$ be an abelian Lie group and $\operatorname{Aff}(A)$ denote the group of affine automorphisms (namely composites of translations and continuous automorphisms) of $A$. We view $A$ as a subgroup of $\operatorname{Aff}(A)$ consisting of the corresponding translations. It can be seen that the above theorem means that a probability measure on $A$ which is infinitely divisible as a measure on $\operatorname{Aff}(A)$ is already infinitely divisible on $A$ itself (see Lemma 2.2). We shall also prove the following analogous result

Received July 10, 2002. 
in a more general set up. Here and in the sequel for a probability measure $\mu$ on a locally compact group $G$ we denote by $Z(\mu)$ the centraliser of the support of $\mu$, namely the subgroup consisting of all elements of $G$ commuting with every element of the support of $\mu$. By a characteristic subgroup of a topological group we mean a subgroup invariant under all continuous automorphisms of the group.

Corollary 1.2. Let $G$ be a locally compact group and $A$ be a closed abelian subgroup of $G$. Let $\mu$ be a probability measure on $G$ which is supported on $A$ and infinitely divisible on $G$. Suppose also that one of the following conditions is satisfied:

i) A is a vector group, namely topologically isomorphic to $\mathbb{R}^{n}$ for some $n$, or

ii) there exists a characteristic closed abelian subgroup $H$ of $Z(\mu)$ such that $H$ is topologically isomorphic to a connected Lie group and contains $A$, or

iii) $G$ is a Lie group, $A$ is connected, and there is no proper closed subgroup of $A$ containing the support of $\mu$.

Then $\mu$ is infinitely divisible on $A$.

A probability measure $\mu$ on a locally compact group is said to be embeddable if there exists a continuous one-parameter convolution semigroup $\left\{\mu_{t}\right\}_{t \geq 0}$ such that $\mu_{1}=\mu$. The question of embeddability of infinitely divisible probability measures, the so-called 'embedding problem', has been studied in literature to a considerable extent (see [4] and [8] for some details and other references). In this respect we note that since every infinitely divisible probability measure on any compactly generated abelian Lie group is embeddable (see [7], $\S$ 3.5.11), Corollary 1.2 implies the following.

Corollary 1.3. Let $G, \mu$ and $A$ be as in Corollary 1.2. Then $\mu$ is embeddable on $A$, and hence also on $G$.

The proof of Theorem 1.1 is achieved by first proving that if $G$ be the semidirect product of a locally compact compactly generated abelian group $A$ with the circle group $T$, and $\mu$ be a probability measure supported on $A$ and infinitely divisible on $G$ then either $\mu$ is infinitely divisible on $A$ or there exists a natural number $n$ such that there is no root of $\mu$ supported on a coset of the form $A t$ for any $t \in T$ of order $n$ (see Corollary 4.2). Theorem 1.1 is deduced from this using some results on embeddability of infinitely divisible measures, namely the embedding theorem from [3] for the case when $A$ is a vector space, and a recent result from [5] for the general case (see $\S 5$ ). Corollary 1.2 is deduced from Theorem 1.1 in $\S 5$ using standard facts about roots of probability measures and automorphism groups of abelian Lie groups.

It may be seen that the conclusion as in Corollary 1.2 does not hold in general for subgroups $A$ which are not connected; e.g. point measures on $\mathbb{Z}$ at nonzero points are infinitely divisible on $\mathbb{R}$ but not on $\mathbb{Z}$. It would be interesting to know of other conditions under which the analogous conclusion does hold. 


\section{Preliminaries}

In this section we set up some notation and recall some facts needed in the sequel.

For any locally compact group $G$ we shall denote by $P(G)$ the space of all probability measures on $G$ equipped with the usual weak* topology and the convolution product. For simplicity we shall denote the convolution product by simple juxtaposition (dropping $*$ ). The unit point masses will be denoted by the corresponding element in the support. If $H$ is a closed subgroup of $G$ we identify $P(H)$ canonically with the subset of $P(G)$ consisting of measures whose support is contained in $H$.

For a locally compact group $G$ we denote by Aut $(G)$ the group of all bicontinuous automorphisms of $G$, and by $\operatorname{Aff}(G)$ the group of all affine automorphisms (namely composites of translations and bicontinuous automorphisms) of $G$. The group $G$ will be realised as a subgroup of $\operatorname{Aff}(G)$ consisting of the corresponding left translations. When $G$ is a Lie group $\operatorname{Aut}(G)$ and $\operatorname{Aff}(G)$ also carry Lie group structures, and we shall consider them equipped with these.

As usual $\mathbb{I N}$ will denote the set of natural numbers and $\mathbb{R}$ the topological group of real numbers. By a vector group we mean a topological group topologically isomorphic to $\mathbb{R}^{n}$ for some $n \in \mathbb{N}$, and by a vector subgroup of a locally compact group we mean a closed subgroup which is a vector group. The vector groups will be considered with the canonical vector space structure, and we note that with respect to it all continuous automorphisms are linear.

Lemma 2.1. Let $V$ be a vector group. Let $K$ be a compact subgroup of Aut $(V)$ and let $\left\{a_{i}\right\}$ be a sequence in $K$ converging to an element a of $K$ such that the a-action on $V$ has no nonzero fixed point. Let $\left\{\lambda_{i}\right\}$ be a sequence in $P(V)$ such that for each $i, \lambda_{i}$ is $a_{i}$-invariant. Suppose there exists a sequence $\left\{v_{i}\right\}$ in $V$ such that $\left\{v_{i} \lambda_{i}\right\}$ is relatively compact. Then $\left\{\lambda_{i}\right\}$ and $\left\{v_{i}\right\}$ are relatively compact.

Proof. We consider $V$ equipped with a norm $\|\cdot\|$ on $V$ invariant under the action of $K$. As $\left\{v_{i} \lambda_{i}\right\}$ is relatively compact there exists a closed ball $B$ (with respect to that norm) such that $v_{i} \lambda_{i}(B) \geq 2 / 3$ for all $i$. Therefore $\lambda_{i}\left(v_{i}^{-1} B\right) \geq 2 / 3$ for all $i$. As $\lambda_{i}$ is $a_{i}$-invariant for each $i$ and $B$ is $K$-invariant we get also that $\lambda_{i}\left(a_{i}\left(v_{i}^{-1}\right) B\right) \geq 2 / 3$ for all $i$. Hence $a_{i}\left(v_{i}^{-1}\right) B \cap v_{i}^{-1} B$ is nonempty for all $i$. Therefore $\left\{a_{i}\left(v_{i}\right) v_{i}^{-1}\right\}$ is contained in a compact set, namely $B B^{-1}$. In the vector space (additive) notation on $V$ this means that $\left\{a_{i}\left(v_{i}\right)-v_{i}\right\}$ is bounded. Suppose $\left\{v_{i}\right\}$ is unbounded. Then passing to a subsequence we may assume that $\left\|v_{i}\right\| \rightarrow \infty$ as $i \rightarrow \infty$. For each $i$ let $w_{i}=v_{i} /\left\|v_{i}\right\|$. Then $\left\{w_{i}\right\}$ is consists of elements of norm 1 , and hence has a nonzero limit point, say $w$. Since $\left\{a_{i}\left(v_{i}\right)-v_{i}\right\}$ is bounded and $\left\|v_{i}\right\| \rightarrow \infty$, we get that $\left(a_{i}\left(w_{i}\right)-w_{i}\right) \rightarrow 0$ as $i \rightarrow \infty$. Since $a_{i} \rightarrow a$ and $w$ is a limit point of $\left\{w_{i}\right\}$ it follows that $a(w)=w$, contradicting the condition in the hypothesis that the $a$-action has no nonzero fixed point. Therefore $\left\{v_{i}\right\}$ is relatively compact. As $\left\{v_{i} \lambda_{i}\right\}$ is relatively compact this implies also that $\left\{\lambda_{i}\right\}$ is relatively compact. This proves the lemma. 
We next describe some simple properties of convolution roots of probability measures.

Lemma 2.2. Let $G$ be a locally compact group and $H$ be a closed normal subgroup of $G$. Let $\mu \in P(H)$. Then we have the following.

i) If $\nu \in P(G)$ is a $k$-th root of $\mu$ for some $k$, then $\nu$ can be expressed as $\lambda$ a, with $\lambda \in P(H)$ and $a \in G$ such that $a^{k} \in H$.

ii) If $G$ is the semidirect product of $H$ and a locally compact group $R$ acting continuously as a group of automorphisms of $H$, then for $\lambda \in P(H)$ and $a \in R$ the measure $\lambda a$ is a $k$-th root of $\mu$ if and only if $a^{k}=e$, the identity element, and $\lambda a(\lambda) \cdots a^{k-1}(\lambda)=\mu$.

iii) If $G, H$ and $R$ are as in (ii) and, furthermore, $H$ is abelian and $a \in R$ is such that $\lambda a$ is a $k$-th root of $\mu$ for some $\lambda \in P(H)$ and $k \geq 1$, then $\mu$ is invariant under the action of $a$ on $H$.

Proof. If $\nu \in P(G)$ and $\nu^{k}=\mu$, and $\eta: G \rightarrow G / H$ is the canonical projection homomorphism, then $\eta(\nu)^{k}=\eta(\mu)$ which is the point mass at the identity element in $G / H$. Hence $\eta(\nu)$ is a point mass in $G / H$ and therefore $\nu$ can be expressed as $\lambda a$, with $\lambda \in P(G)$ and $a \in G$. Also, $\eta(a)^{k}=\eta(\lambda a)^{k}=\eta(\mu)$ is the identity element, and hence $a^{k} \in H$. This proves (i).

Now let $G$ be a semidirect product of $H$ and $R$ as in the hypothesis of (ii) and (iii). Let $\lambda \in P(H)$ and $a \in R$. A straightforward computation shows that $(\lambda a)^{k}=\lambda a(\lambda) \cdots a^{k-1}(\lambda) a^{k}$, which readily implies assertion (ii). Also, if $H$ is abelian and $\lambda \in P(G)$ and $a \in R$ are such that $a^{k}=e$ and $\mu=\lambda a(\lambda) \cdots a^{k-1}(\lambda)$ then we have

$$
a(\mu)=a(\lambda) a^{2}(\lambda) \cdots a^{k-1}(\lambda) \lambda=\lambda a(\lambda) \cdots a^{k-1}(\lambda)=\mu,
$$

which proves the last assertion.

We note that given a connected abelian Lie group $A$, assertions (ii) and (iii) above apply in particular to $G=\operatorname{Aff}(A), H=A$ and $R=\operatorname{Aut}(A)$.

\section{Actions of compact Lie groups}

In this section we describe a decomposition of locally compact abelian groups with respect to actions of a compact connected Lie group (see Theorem 3.3 below). We shall freely use results from the duality theory for locally compact abelian groups; the reader is referred to [6] for general reference in this respect. We begin the following Lemma which is quite classical in flavour.

Lemma 3.1. Let $B$ be a locally compact abelian group containing a compact subgroup $C$ such that $B / C$ is a vector group. Let $K$ be a compact connected group acting continuously as a group of automorphisms of $B$. Let $F$ be the subgroup of $B$ consisting of all elements fixed under the $K$-action. Then there exists a $K$-invariant vector subgroup $W$ of $B$ such that $B$ is the direct product of $F$ and $W$. 
Proof. Let $X=\hat{B}$, the character group of $B$; for convenience we shall use the additive notation for the group $X$. The $K$-action on $B$ induces a $K$-action on $X$. We note that $X^{0}$, the connected component of the identity in $X$, is a vector subgroup invariant under the $K$-action, and $X / X^{0}$ is the discrete character group of $C$. Let $Y$ be the subgroup of $X$ consisting of all elements fixed under the $K$-action. In the light of the Pontryagin duality theorem to prove the lemma it suffices to show that there exists a $K$-invariant vector subgroup $H$ of $X$ such that $X$ is the direct product of $H$ and $Y$. The factor $K$-action on $X / X^{0}$ is trivial and hence for each $x \in X$ and $k \in K, k(x)-x \in X^{0}$. For each $x \in X$ let $f(x)=x+\int_{K}(k(x)-x) \mathrm{d} k$, the integral being with respect to the normalised Haar measure on the compact group $K$. Then $f$ is a continuous homomorphism of $X$ into itself. Let $H$ be the kernel of $f$. For any $x \in X$ and $k_{0} \in K$ we have $k_{0}(f(x))=k_{0}(x)+\int_{K} k_{0}(k(x)-x) \mathrm{d} k=x+\int_{K}\left(k_{0}(x)-x\right) \mathrm{d} k+\int_{K} k_{0}(k(x)-$ $x) \mathrm{d} k=x+\int_{K}\left(k_{0} k(x)-x\right) \mathrm{d} k=f(x)$. Thus $f(x) \in Y$ for all $x \in X$. Also clearly $f(y)=y$ for all $y \in Y$, and in particular $f^{2}(x)=f(x)$ for all $x \in X$. Therefore $H \cap Y$ is trivial and $x-f(x) \in H$ for all $x \in X$. As each $x \in X$ can be expressed as $(x-f(x))+f(x)$, this shows that $X$ is the direct product of $H$ and $Y$. This proves the lemma.

We note also the following.

Lemma 3.2. Let $A$ be a locally compact abelian group and $K$ be a compact group acting continuously on $A$ by automorphisms. Let $B$ be a closed subgroup of $A$ such that $k(b)=b$ for all $b \in B$ and $k \in K$. If $x \in A$ is such that $k(x) \in x B$ for all $k \in K$ and $\psi_{x}: K \rightarrow B$ is the map defined by $\psi_{x}(k)=x^{-1} k(x)$ for all $k \in K$, then $\psi_{x}$ is a continuous homomorphism of $K$ into $B$. If $B$ is a vector group and the factor action of $K$ on $A / B$ is trivial then the $K$-action on $A$ is trivial.

Proof. Let $x \in A$ be such that $k(x) \in x B$ for all $k \in K$ and $\psi=\psi_{x}$, in the notation as above. Then $\psi\left(k_{1} k_{2}\right)=x^{-1} k_{1}\left(k_{2}(x)\right)=x^{-1} k_{1}\left(x \psi\left(k_{2}\right)\right)=$ $x^{-1} k_{1}(x) k_{1}\left(\psi\left(k_{2}\right)\right)=\psi\left(k_{1}\right) \psi\left(k_{2}\right)$, as $\psi\left(k_{2}\right) \in B$ and hence fixed by $k_{1}$. This shows that $\psi=\psi_{x}$ is a homomorphism. Clearly it is continuous.

Now suppose that $B$ is a vector group and that the factor action of $K$ on $A / B$ is trivial. Let $x \in A$ be arbitrary. Then $k(x) \in x B$. Let $\psi_{x}: K \rightarrow B$ be the map as above. Since $\psi_{x}$ is a continuous homomorphism, $\psi_{x}(K)$ is a compact subgroup of $B$, and as the latter is a vector group $\psi_{x}(K)$ is trivial. Hence $k(x)=x$ for all $k \in K$. This proves the lemma.

Theorem 3.3. Let $A$ be a locally compact abelian group and let $K$ be a compact connected Lie group acting continuously on A as a group of automorphisms. Let $e$ be the identity element of $A$. Then there exist closed $K$-invariant subgroups $N$ and $W$ of $A$ such that the following conditions are satisfied:

i) $N \cap W=\{e\}$ and $A=N W$;

ii) $W$ is a vector group and the action of $K$ on $W$ has no nonzero fixed point;

iii) $k(x)=x$ for all $k \in[K, K]$ and $x \in N$; 
iv) If, for $k \in K, \varphi_{k}: A \rightarrow A$ is the homomorphism defined by $\varphi_{k}(x)=$ $x^{-1} k(x)$ for all $x \in A$ then $\varphi_{k_{1}} \varphi_{k_{2}}(x)=e$ for all $k_{1}, k_{2} \in K$ and $x \in N$.

Proof. Let $\Omega_{0}$ be a compact neighbourhood of $e$ in $A$ invariant under the action of $K$. Let $B_{0}$ be the subgroup of $A$ generated by $\Omega_{0}$. Then $B_{0}$ is a compactly generated abelian subgroup which is open in $A$. Let $C$ be the (unique) maximal compact subgroup of $B_{0}$. Let $B$ be the closed subgroup of $B_{0}$ containing $C$ and such that $B / C$ is the connected component of the identity in $B_{0} / C$. Then $C$ and $B$ are invariant under the action of $K$. Furthermore, as $C$ is a compact abelian group its character group is discrete, and since $K$ is connected it follows that the $K$-action on $C$ is trivial. We note also that $B / C$ is a vector group, as it is the connected component of the identity in $B_{0} / C$.

Now let $F$ be the subgroup of $B$ consisting of all elements fixed under the action of $K$. Then by Lemma 3.1 there exists a $K$-invariant vector subgroup $W$ of $B$ such that $F \cap W=\{e\}$ and $B=F W$. For each $k \in K$ let $\varphi_{k}$ be the homomorphism $x \mapsto x^{-1} k(x)$ for all $x \in A$ (as in the statement of the theorem). Let $N$ be the subgroup consisting of all $x$ in $A$ such that $\varphi_{k}(x) \in F$ for all $k \in K$. Then $N$ is a closed subgroup containing $F$. Also, for any $x \in N$ and $h, k \in K$ we have $\varphi_{k}(h(x))=\varphi_{k}\left(x \varphi_{h}(x)\right)=\varphi_{k}(x) \varphi_{k}\left(\varphi_{h}(x)\right)=\varphi_{k}(x)$, as $\varphi_{h}(x) \in F$, and hence $\varphi_{k}\left(\varphi_{h}(x)\right)=e$. This shows that $N$ is $K$-invariant.

We shall show that the assertions as in the theorem hold for the choices $N$ and $W$ as above. Note that statement (ii) is immediate from the choice of $W$. Also, assertion (iv) follows directly from the condition that $\varphi_{k}(x) \in F$ for all $x \in N$ and $k \in K$. Now let $x \in N$. Then $x^{-1} k(x)=\varphi_{k}(x) \in F$ for all $k \in K$. Since the $K$-action on $F$ is trivial, by Lemma 3.2 we get that $k \mapsto \varphi_{k}(x)$ is a homomorphism of $K$ into $F$. Since $F$ is commutative, it follows that $\varphi_{k}(x)=e$ for all $k \in[K, K]$. Therefore $k(x)=x$ for all $k \in[K, K]$. This proves (iii). It remains to prove (i).

As $B$ is open in $A, A / B$ is discrete and hence the $K$-action on $A / B$ is trivial. Also the choice of $W$ shows that the $K$-action on $B / C W$ is trivial. Since $B / C W$ is a vector group, by Lemma 3.2 these observations imply that the $K$-action on $A / C W$ is trivial. Therefore $\varphi_{k}(A)$ is contained in $C W$ for all $k \in K$. Hence for all $x$ in $N \varphi_{k}(x) \in F \cap C W=C$ for all $k \in K$. This implies that the $K$-action on $N / C$ is trivial. On the other hand the $K$-action on $W C / C$ is equivalent to that on $W$, and hence has no nontrivial fixed point. Therefore the intersection of $N / C$ and $W C / C$ in $A / C$ is the trivial subgroup. Since $C$ is contained in $N$ and intersects $W$ trivially, this shows that $N \cap W$ is trivial. This proves the first part of (i).

Now let $x \in A$ be given. We shall show that $x \in N W$. Let $H$ be the smallest closed subgroup containing $C W$ and $x$. Since, as seen above, the $K$-action on $A / C W$ is trivial, it follows that $H$ is $K$-invariant. Clearly $H$ is compactly generated. Let $D$ be the maximal compact subgroup of $H$ (which may be larger than $C$ ). Then $D$ is $K$-invariant, and furthermore the $K$-action on $D$ is trivial. Therefore $D$ is contained in $N$. We note that $H / C W$ has a dense cyclic subgroup and hence it is either compact or infinite cyclic. If it is compact then $H$ is a 
direct product of $D$ and $W$, and hence we get that $x \in D W \subset N W$. Now suppose that $H / C W$ is infinite cyclic. Then $H / C$ is a direct product of $C W / C$ and the infinite discrete cyclic subgroup generated by $x C$. Therefore $H / C$ can be realised as a subgroup of a vector space, say $V$, such that $C W / C$ corresponds to a hyperplane in $V$ and $x C$ to a point outside the hyperplane. The $K$-action on $H / C$ extends to a linear $K$-action on $V$. Since the hyperplane $C W / C$ is $K$-invariant, and $K$ is compact, $V$ contains a line (one-dimensional subspace), say $L$, complementary to $C W / C$ which is pointwise fixed under the $K$-action. This shows that the $K$-action on $H / C$ has a fixed point contained in $x C W / C$. Thus there exists $y \in x C W$ such that $k(y) \in y C$, for all $k \in K$. Thus $\varphi_{k}(y) \in C$ for all $k \in K$ and hence $y \in N$. Since $y \in x C W$, we now get $x \in y C W \subset N W$. This shows that $A=N W$; this completes the proof of assertion (i) and hence of the theorem.

It may be worthwhile to note the following consequence of Theorem 3.3.

Corollary 3.4. Let $A$ be a locally compact abelian group and let $K$ be a compact connected semisimple Lie group acting continuously on $A$ as a group of automorphisms. Then $A$ is the direct product of a closed $K$-invariant subgroup $N$ pointwise fixed under the $K$-action, and a vector group $W$.

Proof. This is immediate from the theorem and the fact that for a semisimple Lie group $K,[K, K]=K$.

Remark 3.5. In general the action on the $N$-component as in Theorem 3.3 can be nontrivial, as can be seen from the following example. Let $T$ be the circle group $A=T \times \mathbb{Z}$, the direct product of $T$ with the group of integers. There is a $T$-action on $A$ defined by $a((t, n))=\left(a^{n} t, n\right)$ for all $a, t \in T$ and $n \in \mathbb{Z}$. For this action the subgroup $N$ as in Theorem 3.3 is the whole of $A$, and the $T$-action on it nontrivial.

\section{Infinite divisibility}

Let $A$ be a locally compact compactly generated abelian group. In this section we consider infinite divisibility of measures on $A$ in a semidirect product of $A$ with the circle group (the multiplicative group of complex numbers of modulus 1 ), and also with certain other Lie groups.

Let $T$ denote the circle group and suppose that a continuous action of $T$ on $A$, as a group of automorphisms of $A$, is given. Let $Q$ be the semidirect product of $A$ and $T$ with respect to the action. Let $\eta: Q \rightarrow T$ be the projection homomorphism. For a measure $\nu$ such that $\eta(\nu)$ is a point mass we shall also denote by $\eta(\nu)$ the corresponding point of support. We now prove the following theorem which provides a way of obtaining roots for a probability measure on $A$, from a sequence of roots on $Q$.

Theorem 4.1. Let the notation be as above. Let $\mu \in P(A)$ and $k \in \mathbb{N}$. For any $p \in \mathbb{N}$ let $d(p)$ be the smallest divisor of $p$ such that $p / d(p)$ is coprime to $k$. 
Suppose that there exists a family $\left\{\nu_{p}\right\}_{p \in \Phi}$ of probability measures on $Q$ indexed by a subset $\Phi$ of $\mathbb{N}$, such that $\nu_{p}^{k p}=\mu$ for all $p$ and $\left\{\eta\left(\nu_{p}\right)^{d(p)} \mid p \in \Phi\right\}$ is an infinite subset of $T$. Then $\mu$ has a $k$-th root on $A$ and, furthermore, it can be chosen to be invariant under the T-action on $A$.

Proof. For convenience the proof is divided into three steps.

\section{A selection of $k$-th roots}

For families indexed by the set $\Phi$ as in the hypothesis we shall suppress $\Phi$ from the notation and write as $\left\{\nu_{p}\right\}$ for $\left\{\nu_{p}\right\}_{p \in \Phi}$ etc. By Lemma 2.2 each $\nu_{p}$, $p \in \Phi$, can be expressed as $\nu_{p}=\lambda_{p} t_{p}$ where $\lambda_{p} \in P(A)$ and $t_{p}=\eta\left(\nu_{p}\right) \in T$. For each $p \in \Phi$ we now define $\sigma_{p} \in P(A)$ as follows: Let $d=d(p)$ and $q=p / d$. Let

$$
\lambda_{p}^{\prime}=\lambda_{p} t_{p}\left(\lambda_{p}\right) \cdots t_{p}^{d-1}\left(\lambda_{p}\right) \text { and } \sigma_{p}=\lambda_{p}^{\prime} t_{p}^{d k}\left(\lambda_{p}^{\prime}\right) \cdots t_{p}^{(q-1) d k}\left(\lambda_{p}^{\prime}\right) .
$$

Then $\left(\sigma_{p} t_{p}^{p}\right)^{k}=\sigma_{p} t_{p}^{p}\left(\sigma_{p}\right) \cdots t_{p}^{(k-1) p}\left(\sigma_{p}\right)$, and the latter is the product of all the measures $t_{p}^{\alpha p+\beta d k+\gamma}\left(\lambda_{p}\right)$, with $0 \leq \alpha \leq k-1,0 \leq \beta \leq q-1$ and $0 \leq \gamma \leq d-1$; the order does not matter in the product, as the group is commutative. From the fact that $p / d$ and $k$ are coprime it follows that no two integers from the set $\{\alpha p+\beta d k+\gamma \mid 0 \leq \alpha \leq k-1,0 \leq \beta \leq q-1,0 \leq \gamma \leq d-1\}$ are congruent to each other modulo $k p$. Since $t_{p}^{k p}=1$ it follows that the product of $t_{p}^{\alpha p+\beta d k+\gamma}\left(\lambda_{p}\right)^{\text {'s }}$ as above is the same as $\lambda_{p} t_{p}\left(\lambda_{p}\right) \cdots t_{p}^{(k p-1)}\left(\lambda_{p}\right)$, and since $\nu_{p}$ is a $k p$-th root it is precisely $\mu$. Thus $\sigma_{p} t_{p}^{p}$ is a $k$-th root of $\mu$. We note also that each $\sigma_{p}$ is $t_{p}^{d(p) k}$-invariant.

For each $p \in \Phi, t_{p}^{p}$ is a $k$-th root of 1 in $T$. The set of these roots is finite, and hence there exists a $k$-th root of 1 in $T$, say $s$, such that $\left\{\eta\left(\nu_{p}\right)^{d(p)} \mid p \in \Phi, t_{p}^{p}=s\right\}$ is infinite. Therefore replacing $\Phi$ by $\left\{p \in \Phi \mid t_{p}^{p}=s\right\}$ we may without loss of generality assume that $t_{p}^{p}=s$ for all $p \in \Phi$. Thus for each $p \in \Phi, \sigma_{p} s$ is a $k$-th root of $\mu$, which is $t_{p}^{d(p) k}$-invariant.

\section{Relative compactness}

Since each root is a two sided factor, by the shift compactness theorem (see [9], Ch. III, Theorem 2.2) it follows that there exist $\left\{x_{p}\right\}$ in $Q$ such that $\left\{x_{p} \sigma_{p} s\right\}$ is relatively compact. Since $Q / A$ is compact $\left\{x_{p}\right\}$ can be chosen to be contained in $A$. Let $A=N W$ be the decomposition of $A$ as in Theorem 3.3 with respect to the action of $T$ as above (in the place of $K$ in the theorem). Since $W$ is $T$-invariant it is a normal subgroup of $Q$. Let $\pi: Q \rightarrow Q / W$ be the quotient homomorphism. The group $Q / W$ can be identified canonically with the subgroup $N T$. For $t \in T$ let $\varphi_{t}$ be the map as before, defined by $\varphi_{t}(x)=x^{-1} t(x)=x^{-1} t x t^{-1}$ for all $x \in A$. Recall that by condition (iv) of Theorem $3.3 \varphi_{t_{1}} \varphi_{t}(x)=e$ for all $t$ and $t_{1}$ in $T$, and $x \in N$. This means that, for any $t \in T, \varphi_{t}(N)$ is centralised by $T$ and hence contained in the center of $N T$. It follows that $[N T, N T]$ is contained in the center of $N T$. Therefore $N T$ is a nilpotent group. Thus $Q / W$ is a nilpotent group. Since $\left\{x_{p} \sigma_{p} s\right\}$ is relatively compact, $\left\{\pi\left(x_{p}\right) \pi\left(\sigma_{p} s\right)\right\}$ is relatively compact. On the other hand $\left\{\pi\left(\sigma_{p} s\right)\right\}$ are all roots of $\pi(\mu)$, and since $Q / W$ is a compactly 
generated nilpotent group this implies that $\left\{\pi\left(\sigma_{p} s\right)\right\}$ is relatively compact (see [7], Theorems 3.1.17 and 3.1.13). Hence $\left\{\pi\left(x_{p}\right)\right\}$ is relatively compact.

Now let $\psi: Q \rightarrow Q / N$ be the quotient homomorphism onto $Q / N$. The latter can be realised canonically as the semidirect product of $W$ and $T$ under the action of $T$ on $W$. Since $\left\{x_{p} \sigma_{p} s\right\}$ is relatively compact, $\left\{\psi\left(x_{p}\right) \psi\left(\sigma_{p}\right)\right\}$ is relatively compact. Recall that $\sigma_{p}$ is $t_{p}^{d(p) k}$-invariant. Hence the measure $\psi\left(\sigma_{p}\right)$ on $W$ is invariant under the action of $t_{p}^{d(p) k}$. For each $p$ let $F_{p}$ be the subgroup generated by $t_{p}^{d(p) k}$. Then $\psi\left(\sigma_{p}\right)$ is in fact $F_{p}$-invariant for each $p$. As $t_{p}=\eta\left(\nu_{p}\right)$, by hypothesis $\left\{t_{p}^{d(p)}\right\}$ is an infinite subset of $T$, and hence so is $\left\{t_{p}^{d(p) k}\right\}$. Therefore given any $a \in T$ we can find $a_{p} \in F_{p}$, for all $p \in \Phi$, such that $\left\{a_{p}\right\}$ converges to $a$; we choose $a$ to be an element generating a dense subgroup of $T$ and $\left\{a_{p}\right\}$ to be a family satisfying this condition. Now we have the measures $\psi\left(\sigma_{p}\right)$ invariant under $a_{p}$ for each $p$, with $a_{p} \rightarrow a$, and $\psi\left(x_{p}\right) \in W$ such that $\left\{\psi\left(x_{p}\right) \psi\left(\sigma_{p}\right)\right\}$ is relatively compact. As the $T$-action on $W$ has no nontrivial fixed point on $W$, and the subgroup generated by $a$ is dense in $T$, the $a$-action on $W$ also has no nontrivial fixed point on $W$. Therefore by Lemma 2.1 it follows that $\left\{\psi\left(x_{p}\right)\right\}$ is relatively compact.

Since $A=N W$, a direct product, and $\left\{\pi\left(x_{p}\right)\right\}$ and $\left\{\psi\left(x_{p}\right)\right\}$ are relatively compact, it follows that $\left\{x_{p}\right\}$ is relatively compact. Since by choice $\left\{x_{p} \sigma_{p} s\right\}$ is relatively compact we now get that $\left\{\sigma_{p} s\right\}$ is relatively compact.

3. Completion of the proof.

Let $\sigma$ be a limit point of $\left\{\sigma_{p}\right\}$, which by Step 2 is relatively compact. Let $\left\{a_{p}\right\}$ be as in Step 2. Since $a_{p} \rightarrow a$ and $\sigma_{p}$ is $a_{p}$-invariant we get that $\sigma$ is $a$-invariant. Since $a$ generates a dense subgroup of $T$ it follows that $\sigma$ is invariant under the action of $T$, and in particular under the action of $s$. Now since $\sigma s$ is a limit point of $\sigma_{p} s$ which are $k$-th roots of $\mu$ it follows that $(\sigma s)^{k}=\mu$. As $\sigma$ is $s$-invariant this implies that $\sigma^{k}=\mu$. Thus $\mu$ has a $k$-th root on $A$ which is invariant under the action of $T$. This proves the theorem.

Corollary 4.2. Let $A$ be a locally compact compactly generated abelian group and let $Q$ be the semidirect product of $A$ with the circle group $T$, with respect to a continuous action of $T$ on $A$ by automorphisms. Let $\eta: Q \rightarrow T$ be the projection homomorphism. Let $\mu \in P(A)$ and suppose that for every $n \in \mathbb{N}$ there exists a root $\nu$ of $\mu$ (of some order) on $Q$ such that $\eta(\nu)$ is an element of order $n$ in $T$. Then $\mu$ is infinitely divisible on $A$.

Proof. Suppose that $\mu$ is not infinitely divisible on $A$, and let $k \in \mathbb{N}$ be such that $\mu$ has no $k$-th root on $A$. As before, for any $p \in \mathbb{N}$ let $d(p)$ denote the smallest divisor of $p$ such that $p / d(p)$ is coprime to $k$. Consider the set $R=$ $\left\{\eta(\nu)^{d(p)} \mid \nu \in P(Q), p \in \mathbb{N}, \nu^{k p}=\mu\right\}$. Since, by the choice of $k, \mu$ has no $k$-th root on $A$, Theorem 4.1 implies that $R$ is finite. As the elements of $R$ are roots of 1 in $T$ it follows that the subgroup of $T$ generated by $R$ is also finite. Let $m$ be the order of the subgroup generated by $R$. Now let $n$ be a natural number coprime to $\mathrm{km}$. By the condition in the hypothesis there exists a root $\nu$ of $\mu$ 
on $Q$ such that $\eta(\nu)$ is of order $k n$ in $T$; thus $\nu^{k n p}=\mu$ for some $p \in \mathbb{I N}$. Then $\eta(\nu)^{d(n p)} \in R$, and hence $\eta(\nu)^{d(n p) m}=1$. Therefore $k n$, which is the order of $\eta(\nu)$, divides $d(n p) m$. The prime factors of the latter are the same as those of $\mathrm{km}$, and since by choice $n$ is coprime to $\mathrm{km}$, this is a contradiction. Therefore $\mu$ is infinitely divisible on $A$. This proves the corollary.

The corollary can be readily generalised to a slightly more general class of semidirect products. We shall say that a Lie group $S$ is of type $\mathcal{C}$ if $S$ has a circle subgroup $T$ such that every element of finite order in $S$ is conjugate to an element of $T$. We note that any compact connected Lie group of rank 1 is of type $\mathcal{C}$, since in this case any maximal torus is a circle group and all maximal tori are conjugate. The special linear group $S L(2, \mathbb{R})$ is also of type $\mathcal{C}$, so are its quotient $P S L(2, \mathbb{R})$ and all the covering groups with finite fibers. The semidirect product of $T$, the circle group, with a vector group $V$ under an action of $T$ on $V$ can also be seen to be a group of type $\mathcal{C}$. We now extend the result as in Corollary 4.2 for $T$-actions to actions of these groups, under the condition that the measure $\mu$ is invariant under the action.

Corollary 4.3. Let $A$ be a locally compact compactly generated abelian group and $\mu \in P(A)$. Let $S$ be a Lie group of type $\mathcal{C}$ acting continuously as a group of automorphisms preserving $\mu$. Let $Q$ be the semidirect product of $A$ with $S$, with respect to the action and $\eta: Q \rightarrow S$ be the projection homomorphism. Let $\mu \in P(A)$ and suppose that for every $n \in \mathbb{N}$ there exists a root $\nu$ of $\mu$ on $Q$ such that $\eta(\nu)$ is an element of order $n$. Then $\mu$ is infinitely divisible on $A$.

Proof. Let $T$ be a circle subgroup of $S$, such that every element of finite order in $S$ is conjugate to an element of $T$. Let $k \in \mathbb{N}$ and let $\nu$ be a $k$-th root of $\mu$ on $Q$. Then by Lemma 2.2 there exist $\lambda \in P(A)$ and $g \in S$ such that $\nu=\lambda g$ and $g^{k}$ is the identity element. Since $g$ is of finite order there exist $h \in T$ and $x \in S$ such that $h=x g x^{-1}$. Let $\nu^{\prime}=x \nu x^{-1} \in P(A)$. Then $\left(\nu^{\prime} h\right)^{k}=x(\nu g)^{k} x^{-1}=x \mu x^{-1}=\mu$, where the last step follows from the condition that $\mu$ is invariant under the action of $S$ on $A$. Thus $\mu$ has a $k$-th root on $A T$, for $k \in \mathbb{N}$, whenever it has a $k$-th root on $Q$. The corollary now follows from Corollary 4.2 .

\section{Proofs of Theorem 1.1 and Corollary 1.2}

In this section we prove the results stated in the introduction. We first recall the following.

Lemma 5.1. Let $m \in \mathbb{N}$ and $\Gamma_{m}$ be the group of all continuous automorphisms of the m-dimensional torus. Then there exists $r \in \mathbb{N}$ such that for any $\gamma \in \Gamma_{m}$ of finite order, the order divides $r$.

Proof. The group $\Gamma_{m}$ as above is isomorphic to the group $G L(m, \mathbb{Z})$ of all integral matrices with determinant \pm 1 . For the latter group the property as above is well known (see [10], LG Appendix, for instance). 
Lemma 5.2. Let $G$ be a locally compact group and $H$ be a closed normal subgroup of $G$. Suppose that there exists $r \in \mathbb{N}$ such that the order of any element of finite order in $G / H$ divides $r$. Let $\mu \in P(H)$ and suppose that it is infinitely divisible on $G$. Then $\mu$ is infinitely divisible on $H$.

Proof. Consider any $j \geq 2$ and $\nu$ be a $j r$-th root of $\mu$ on $G$. Let $\eta: G \rightarrow G / H$ be the canonical quotient homomorphism. Then $\eta(\nu)$ is a point mass at a point $a \in G / H$ of finite order. By the condition in the hypothesis the order of $a$ divides $r$ and hence $a^{r}$ is the identity. Therefore $\nu^{r}$ is supported on $H$. Since $\nu$ is a $j r$-th root of $\mu$ it follows that $\nu^{r}$ is a $j$-th root of $\mu$, on $H$. Hence $\mu$ is infinitely divisible on $H$.

Proof of Theorem 1.1. First, consider the case when $A$ is a vector group; we shall denote it as $V$. Let $\mu \in P(V)$ be affinely infinitely divisible. Then $\mu$ is infinitely divisible on $\mathrm{Aff}(V)$. By the embedding theorem of [3] (see also [4]) $\mu$ is embeddable in Aff $(V)$. Thus there exists a continuous one-parameter convolution semigroup $\left\{\mu_{t}\right\}_{t \geq 0}$ in $P(\operatorname{Aff}(V))$ such that $\mu_{1}=\mu$. Let $\eta: \operatorname{Aff}(V) \rightarrow$ $G L(V)$ be the canonical projection. Then $\eta\left(\left\{\mu_{t}\right\}_{t \geq 0}\right)$ is a convolution semigroup of probability measures such that $\eta\left(\mu_{1}\right)$ is the point mass at the identity. It follows that each $\eta\left(\mu_{t}\right), t \geq 0$, is a point measure, say at the point $a_{t} \in G L(V)$. As $a_{1}=I$, the identity automorphism, for negative $t$ we can set $a_{t}=a_{t+m}$ for some (any) integer $m>-t$. Then $\left\{a_{t}\right\}_{t \in \mathbb{R}}$ is a periodic one-parameter subgroup of $G L(V)$. Let $T=\left\{a_{t} \mid t \in \mathbb{R}\right\}$. If $T$ is the trivial subgroup then $\left\{\mu_{t}\right\}$ is contained in $P(V)$ and hence $\mu$ is infinitely divisible on $V$. Suppose $T$ is nontrivial; then it is the circle group. Then for any $n \in \mathbb{N}, \mu$ has a root $\nu$ such that $\eta(\nu)$ is an element of order $n$ in $T$. Hence by Corollary $4.2 \mu$ is infinitely divisible on $V$. This proves the theorem for the case at hand.

Now let $A$ be any connected abelian Lie group, namely $A=\mathbb{T}^{m} \times \mathbb{R}^{n}$ for some $m, n \in \mathbb{N}, \mathbb{T}^{m}$ being the $m$-dimensional torus. Let $\mu \in P(A)$ be affinely infinitely divisible, so $\mu$ is infinitely divisible on $\operatorname{Aff}(A)$. Let $S$ be the maximal torus in $A$. Then $A / S$ is a vector group. Let $B$ be the closed subgroup of $A$ containing $S$, such that $B / S$ is the vector subspace of $A / S$ spanned by $(\operatorname{supp} \mu) S / S$, where $\operatorname{supp} \mu$ denotes the support of $\mu$. We shall show that $\mu$, which can be realised as a measure on $B$ and hence on Aff $(B)$, is infinitely divisible on $\operatorname{Aff}(B)$. Let $k \geq 1$ be given and let $\nu$ be a $k$-th root of $\mu$ on $\operatorname{Aff}(A)$. Then $\nu=\lambda a$, for some $\lambda \in P(A)$ and $a \in \operatorname{Aut}(A)$ such that $a^{k}=I$, the identity element, and $\lambda a(\lambda) \cdots a^{k-1}(\lambda)=\mu$. By Lemma $2.2 \mu$ is $a$-invariant, and hence it follows that $B$ is $a$-invariant. Since $a$ is of finite order, considering the action of $a$ on the Lie algebra of $A$ it can be seen that there exists an $a$-invariant vector subgroup $D$ of $A$ such that $B \cap D$ is trivial and $A=B D$. Let $\pi: A \rightarrow D$ be the projection homomorphism onto $D$. Then $\pi(\mu)$ is the point mass at $e$, the identity element, and since $\lambda a(\lambda) \cdots a^{k-1}(\lambda)=\mu$ it follows that $\pi(\lambda)$ is a point mass at a point $d$ in $D$ such that $d a(d) \cdots a^{k-1}(d)=e$. Then $\lambda d^{-1} \in P(B)$ and $\left(\lambda d^{-1}\right) a\left(\lambda d^{-1}\right) \cdots a^{k-1}\left(\lambda d^{-1}\right)=\lambda a(\lambda) \cdots a^{k-1}(\lambda) d^{-1} a\left(d^{-1}\right) \cdots a^{k-1}\left(d^{-1}\right)=\mu$. This shows that $\mu$ is infinitely divisible on $\operatorname{Aff}(B)$. 
Now let $\Gamma$ be the subgroup of Aut $(B)$ consisting of all automorphisms whose restriction to $S$ is trivial. Then $\operatorname{Aut}(B) / \Gamma$ can be realised as a group of continuous automorphisms of $S$ and hence by Lemma 5.1 there exists $r \in \mathbb{N}$ such that for any element of finite order in $\operatorname{Aut}(B) / \Gamma$ the order divides $r$. Therefore by Lemma $5.2 \mu$ is infinitely divisible on $B \Gamma$.

Let $V$ be a vector subgroup of $B$ such that $B=S V$, direct product. Let $\Theta$ be the group of automorphisms $\theta$ of $B$ of the form $\theta(v) \in S v$ for all $v \in V$. Let $\Delta$ be the subgroup of Aut $(B)$ which leaves $V$ invariant. By writing the matrix forms of the derivatives of the automorphisms it can be verified that $\Gamma=\Theta \Delta$. It may be noted that $\Delta$ is canonically isomorphic to $G L(V), \Theta$ is normalised by $\Delta$, and $\Gamma$ is a semidirect product of $\Theta$ and $\Delta$.

Let $\psi: B \rightarrow V$ be the projection homomorphism onto $V$. Let $K^{\prime}$ be the subgroup of $G L(V)$ consisting of all elements leaving $\psi(\mu)$ invariant. Since the support of $\psi(\mu)$ spans $V$ it follows that $K^{\prime}$ is compact (this is classical; see [1] for a general result in this respect). Let $K^{\prime}$ also denote the subgroup of $\Delta$ corresponding to $K^{\prime}$ under the natural correspondence of $\Delta$ and $G L(V)$. Let $K$ be the connected component of the identity in $K^{\prime}$. If $\nu=\lambda \gamma$ is a root of $\mu$ on $B \Gamma$, where $\lambda \in P(B)$ and $\gamma \in \Gamma$, and $\gamma=\theta \delta$ with $\theta \in \Theta$ and $\delta \in \Delta$, then by Lemma $2.2 \mu$ is $\gamma$-invariant and hence $\psi(\mu)$ is $\delta$-invariant, so $\delta \in K^{\prime}$. This shows that $\mu$ is infinitely divisible on $B \Theta K^{\prime}$. Since $K$ is of finite index in $K^{\prime}$, by Lemma 5.2 it follows that $\mu$ is infinitely divisible on $B \Theta K$.

Now let $N=B \Theta$. Then $[N, N]=S$ is a compact subgroup contained in the center of $N$; in particular $N$ is a nilpotent Lie group. Also $N K$ is a semidirect product of $N$ and $K$. By the embedding theorem in [5] any $\mu$ on $N$ which is infinitely divisible on $N K$ is embeddable on $N K$. By an argument as in the special case when $A$ is a vector group, this together with Theorem 4.1 shows that $\mu$ is infinitely divisible on $N$. Recall that $N=B \Theta$, semidirect product. We note also that $B$ is a normal subgroup of $N$ and that $\Theta$, being a vector subgroup, has no nontrivial elements of finite order. As $\mu \in P(B)$ and infinitely divisible on $N$, this implies that $\mu$ is infinitely divisible on $B$. This proves the theorem.

Proof of Corollary 1.2. Let $G, \mu$, and $A$ be as in the hypothesis. Let $B$ be the smallest closed subgroup of $G$ containing the support of $\mu$. We recall that any root $\nu$ of $\mu$ on $G$ is supported on the normaliser of $B$ in $G$ (see [2], Proposition 1.1). Therefore replacing $G$ by the normaliser of $B$ we may without loss of generality assume that $B$ is normal in $G$.

We shall introduce a further reduction in the case when condition (ii) in the hypothesis is satisfied. Let $H$ be the subgroup of $Z(\mu)$ as in the hypothesis. Note that $Z(\mu)$ is the centraliser of the subgroup $B$ as above. Since by assumption $B$ is normal in $G$, it follows that $Z(\mu)$ is normal in $G$. Since $H$ is a characteristic subgroup of $Z(\mu)$ it follows that $H$ is also normal in $G$. Recall that by hypothesis $H$ is abelian and topologically isomorphic to a connected Lie group. Let $C$ be the (unique) maximal torus in $H$. Then $C$ is normal in $G$. Consider the homomorphism $\phi: G \rightarrow$ Aut $(C)$ defined by $\phi(g)(x)=g x g^{-1}$ for all $g \in G$ 
and $x \in C$. By Lemma 5.1 there exists a natural number $r$ such that for every element of Aut $(C)$ of finite order the order divides $r$. Hence by Lemma $5.2 \mu$ is infinitely divisible on the kernel of $\phi$. Therefore without loss of generality we may assume the conjugation action of $G$ on $C$ to be trivial, i.e. that $C$ is contained in the center of $G$.

For each $k \geq 1$ let $\nu_{k}$ be a $k$-th root of $\mu$ on $G$. Then there exist $\lambda_{k} \in P(B)$ and $g_{k} \in G$ such that $\nu_{k}=\lambda_{k} g_{k}$ and $g_{k}^{k} \in B$. Let $\rho_{k}$ be the automorphism of $B$ induced by inner conjugation by $g_{k}$.

Now consider the first case. Let $k \geq 1$ be arbitrary. Let $V$ be the vector subspace of $A$ spanned by $B$. Let $v_{k}$ be the unique $k$-th root of $g_{k}^{k}$ in $V$ and let $\lambda_{k}^{\prime}=\lambda_{k} v_{k} \in P(V)$. As $V$ is a vector group and $B$ spans $V$ the automorphism $\rho_{k}$ of $B$ extends to a unique automorphism of $V$, say $\tilde{\rho}_{k}$. Since $\rho_{k}^{k}$ is the identity automorphism of $B$ it follows that $\tilde{\rho}_{k}^{k}$ is the identity automorphism of $V$. Also $v_{k}^{k}=g_{k}^{k}$ is fixed by $\rho_{k}$ and hence by $\tilde{\rho}_{k}$, and so by linearity of $\tilde{\rho}$ we get $\tilde{\rho}_{k}\left(v_{k}\right)=v_{k}$. Now we have $\left(\lambda_{k}^{\prime} \tilde{\rho}_{k}\right)^{k}=\lambda_{k}^{\prime} \tilde{\rho}_{k}\left(\lambda_{k}^{\prime}\right) \cdots \tilde{\rho}_{k}^{k-1}\left(\lambda_{k}^{\prime}\right)=\lambda_{k} v_{k} \tilde{\rho}_{k}\left(\lambda_{k} v_{k}\right) \cdots \tilde{\rho}_{k}^{k-1}\left(\lambda_{k} v_{k}\right)=$ $\lambda_{k} \rho_{k}\left(\lambda_{k}\right) \cdots \rho_{k}^{k-1}\left(\lambda_{k}\right) v_{k}^{k}=\lambda_{k} \rho_{k}\left(\lambda_{k}\right) \cdots \rho_{k}^{k-1}\left(\lambda_{k}\right) g_{k}^{k}=\left(\lambda_{k} g_{k}\right)^{k}=\nu_{k}^{k}=\mu$. This shows that $\mu$ is infinitely divisible on Aff $(V)$. Therefore by Theorem $1.1 \mu$ is infinitely divisible on $V$, and hence also on $A$.

Next consider the second case. In this case we first show that $\mu$ is infinitely divisible on Aff $(H)$. Let $k \geq 1$ be arbitrary. Since $H$ is a connected abelian Lie group for each $g_{k}$ as above there exists $h_{k} \in H$ such that $h_{k}^{k}=g_{k}^{k}$. Let $\lambda_{k}^{\prime}=$ $\lambda_{k} h_{k} \in P(H)$. Let $\tilde{\rho}_{k}$ be the automorphism of $H$ induced by inner conjugation by $g_{k}$. As $C$ is contained in the center of $G$ it is pointwise fixed by $\tilde{\rho}_{k}$, and also $h_{k}^{k}=g_{k}^{k}$ is fixed by $\tilde{\rho}_{k}$. Since $H / C$ is a vector group this implies that $\tilde{\rho}_{k}\left(h_{k}\right)=h_{k}$. Now a computation analogous to the one in the first case shows that $\left(\lambda_{k}^{\prime} \tilde{\rho}_{k}\right)^{k}=\mu$. Hence $\mu$ has a $k$-th root on $\operatorname{Aff}(H)$ for all $k \geq 1$. Therefore by Theorem $1.1 \mu$ is infinitely divisible on $H$. Since $\mu \in P(A)$ and $H$ is an abelian Lie group this implies that $\mu$ is infinitely divisible on $A$.

The last case follows as a special case of (ii), where we choose $H$ to be the connected component of the identity in the center of $Z(\mu)$; the condition on $A$ as in the hypothesis ensures that $A$ is contained in the latter. This completes the proof of the corollary.

Remark 5.3. It is not clear whether restrictions such as conditions (i) or (ii) in Corollary 1.2 are indeed necessary for the validity of the conclusion as in the corollary. Pursuing this question has turned out to be too cumbersome for it to be worthwhile for the present.

Remark 5.4. It may be mentioned here that an assertion as in Theorem 1.1 holds for finite-dimensional vector spaces over $p$-adic fields, for any prime $p$, in the place of connected Lie groups. This readily follows from the fact that in the automorphism groups of these groups the orders of elements of finite order are bounded (see [10], LG, Appendix). An analogue of Corollary 1.2 (case (i)) can also be deduced for these groups by a simple argument. 


\section{Acknowledgement}

The work was done when the first-named author was visiting the Erwin Schrödinger Institute, Vienna; the author is grateful for the generous support and hospitality received during the visit.

\section{References}

[1] S. G. Dani, Invariance groups and convergence of types of measures on Lie groups, Math. Proc. Cambridge Philos. Soc. 112 (1992), 91-108.

[2] S. G. Dani, M. McCrudden, Factors, roots and embeddability of measures on Lie groups, Math. Z. 199 (1988), 369-385.

[3] _ Embedding infinitely divisible probability measures on the affine group, Probability measures on groups, IX (Oberwolfach, 1988), 36-49, Lecture Notes in Math., 1379, Springer, Berlin, 1989.

[4] Embeddability of infinitely divisible distributions on linear Lie groups, Invent. Math. 110 (1992), 237-261.

[5] S. G. Dani, M. McCrudden, S. Walker, On the embedding problem for infinitely divisible distributions on certain Lie groups with toral center, preprint.

[6] E. Hewitt, K. A. Ross, Abstract harmonic analysis, Vol. I. Second edition. Grundlehren der Mathematischen Wissenschaften, 115. Springer-Verlag, Berlin-New York, 1979.

[7] H. Heyer, Probability measures on locally compact groups, Ergebnisse der Mathematik und ihrer Grenzgebiete, Band 94. Springer-Verlag, Berlin-New York, 1977.

[8] M. McCrudden, An introduction to the embedding problem for probabilities on locally compact groups, Positivity in Lie theory: open problems, 147-164, de Gruyter Exp. Math., 26, de Gruyter, Berlin, 1998.

[9] K. R. Parthasarathy, Probability measures on metric spaces, Probability and Mathematical Statistics, No. 3 Academic Press, Inc., New York-London, 1967.

[10] J.-P. Serre, Lie algebras and Lie groups, Second edition. Lecture Notes in Mathematics, 1500. Springer-Verlag, Berlin, 1992.

School of Mathematics, Tata Institute of Fundamental Research, Homi Bhabha Road, Colaba, Mumbai 400 005, India.

E-mail address: dani@math.tifr.res.in

Erwin Schrödinger Institute, Boltzmanngasse 9, A-1090 Vienna, Austria.

E-mail address: klaus.schmidt@univie.ac.at 